\title{
On unitary equivalence of quasi-free Hilbert modules
}

by

\author{
Li Chen (Shanghai)
}

\begin{abstract}
We characterize unitary equivalence of quasi-free Hilbert modules, which complements Douglas and Misra's earlier work [New York J. Math. 11 (2005)]. We first confine our arguments to the classical setting of reproducing Hilbert spaces and then relate our result to equivalence of Hermitian vector bundles.
\end{abstract}

1. Introduction. In a 1978 Acta paper ([2]), Cowen and Douglas established the Cowen-Douglas theory, making a connection between operator theory and complex geometry. Since then, various generalizations and applications have been found, and many ideas and methods have been extended to investigation of Hilbert modules ([1], [4], [5], [6]).

The theory of Hilbert modules, introduced by Douglas and Paulsen $([6])$, provides a powerful tool to study multivariate operator theory. For a bounded domain $\Omega$ in $\mathbb{C}^{m}$, let $A(\Omega)$ be the function algebra consisting of functions that are holomorphic in some neighbourhood of $\bar{\Omega}$ with supremum norm. A Hilbert space $R$ is said to be a contractive Hilbert module over $A(\Omega)$ if there exists a module action $A(\Omega) \times R \rightarrow R$ satisfying

$$
\|\varphi \cdot f\|_{R} \leq\|\varphi\|_{A(\Omega)}\|f\|_{R}
$$

for $\varphi$ in $A(\Omega)$ and $f$ in $R$. Two Hilbert modules $R$ and $R^{\prime}$ are said to be isomorphic if there exists a unitary module map from $R$ onto $R^{\prime}$.

Quasi-free Hilbert modules were introduced by Douglas and Misra in the study of resolutions of general Hilbert modules ([4]). It was shown that a large class of Hilbert modules admit resolutions with quasi-free Hilbert modules, therefore quasi-free Hilbert modules can be regarded as building blocks of general Hilbert modules.

A quasi-free Hilbert module can be naturally regarded as a vector-valued reproducing Hilbert space to which one can associate a Hermitian vector bundle. Geometric methods, including curvatures and the Rigidity Theorem, 
play a significant role in obtaining unitary invariants for quasi-free Hilbert modules (see [1], [2], [5]).

To describe unitary equivalence of quasi-free Hilbert modules, Douglas and Misra ([5]) introduced a modulus function $\mu_{\left(R, R^{\prime}\right)}$ for a pair of quasi-free Hilbert modules $R$ and $R^{\prime}$. It is closely related to the geometry of the Hermitian vector bundles associated to the modules; its definition will be given in the next section. Let $\operatorname{Hol}(\Omega)$ be the set of holomorphic matrix-valued functions on $\Omega$. Douglas and Misra gave the following necessary condition for two modules to be unitarily equivalent:

TheOREM 1.1 ([5]). If finite rank quasi-free Hilbert modules $R$ and $R^{\prime}$ over $A(\Omega)$ are unitarily equivalent, then the modulus function $\mu_{\left(R, R^{\prime}\right)}$ is the absolute value of a function $\Psi$ in $\operatorname{Hol}(\Omega)$.

We will mainly consider the converse of Theorem 1.1. In [5], the converse of Theorem 1.1 was proved under the additional hypothesis that the bounded domain $\Omega$ is strongly pseudo-convex. In this paper, by studying the reproducing kernels of the Hilbert modules via calculating the Gramians of certain frames, we are able to show that the condition of Theorem 1.1 is sufficient for any bounded domain $\Omega$. Our main result is as follows:

THEOREM 1.2. If the modulus function $\mu_{\left(R, R^{\prime}\right)}$ for the two finite rank quasi-free Hilbert modules $R$ and $R^{\prime}$ over $A(\Omega)$ is the absolute value of a function $\Psi$ in $\operatorname{Hol}(\Omega)$, then $R$ and $R^{\prime}$ are unitarily equivalent.

The reproducing kernel approach to Cowen-Douglas theory was first adopted by Curto and Salinas ([3]). It enables us to argue without using the language of complex geometry, as will be seen in Section 3 .

In Section 4, we provide a geometric characterization of unitary equivalence. The notion of spectral sheaf will be involved and the proof relies on the calculations made in Section 3. This characterization gives a geometric interpretation of the main theorem.

2. Preliminaries. In this section, we will make a quick review of quasifree Hilbert modules. Throughout the rest of this paper, we use $\left\{e_{1}, \ldots, e_{n}\right\}$ to denote the standard basis of $\mathbb{C}^{n}$.

First we recall some basic elements on localization of Hilbert modules, introduced in [6]. Fix $z$ in $\Omega$. We can construct a one-dimensional Hilbert module $\mathbb{C}_{z}$, which is the one-dimensional Hilbert space $\mathbb{C}$ with module action $\varphi \cdot \xi=\varphi(z) \xi$, for $\varphi$ in $A(\Omega)$ and $\xi$ in $\mathbb{C}$. We denote the complex number 1 by $1_{z}$ in the Hilbert module $\mathbb{C}_{z}$.

Given a Hilbert module $R$ over $A(\Omega)$, the Hilbert module tensor product $R \otimes_{A} \mathbb{C}_{z}$ is called the localization of $R$ at $z$, which is canonically isomorphic to the quotient module $R / R_{z}$ (which in turn is isomorphic to $R_{z}^{\perp}$ with 
compressed module action), where

$$
R_{z}=\left[A(\Omega)_{z} R\right]^{-}, \quad A(\Omega)_{z}=\{\varphi \in A(\Omega) \mid \varphi(z)=0\} .
$$

The isomorphism is given by

$$
R \otimes_{A} \mathbb{C}_{z} \rightarrow R_{z}^{\perp}: f \otimes_{A} 1_{z} \mapsto P_{z} f
$$

where $P_{z}$ denotes the orthogonal projection from $R$ onto $R_{z}^{\perp}$. It is easy to see, by definition of tensor product of Hilbert modules $([6])$, that the module action of a function $\varphi$ in $A(\Omega)$ on $R \otimes_{A} \mathbb{C}_{z}$ is just multiplication by $\varphi(z)$.

Recall that a Hilbert module $R$ is said to be finitely generated if $R$ admits a finite generating set $E$ in the sense that the finite linear sums

$$
\sum_{i=1}^{n} \varphi_{i} \cdot f_{i}, \quad f_{i} \in E, \varphi_{i} \in A(\Omega),
$$

are dense in $R$. The minimal cardinality of a generating set is called the rank of $R$.

Now we give the definition of quasi-free Hilbert modules introduced by Douglas and Misra.

Definition 2.1 ([4]). A finitely generated Hilbert module $R$ of rank $n$ over $A(\Omega)$ is said to be quasi-free relative to the generating set $\left\{f_{1}, \ldots, f_{n}\right\}$ if

(i) $f_{1} \otimes_{A} 1_{z}, \ldots, f_{n} \otimes_{A} 1_{z}$ forms a basis for $R \otimes_{A} \mathbb{C}_{z}$ for every $z$ in $\Omega$,

(ii) the linear map $X_{z}: R \otimes_{A} \mathbb{C}_{z} \rightarrow \mathbb{C}^{n}$ defined by

$$
X_{z}\left(f_{i} \otimes_{A} 1_{z}\right)=e_{i}
$$

is locally uniformly bounded,

(iii) $f \otimes_{A} 1_{z}=0$ for every $z$ in $\Omega$ if and only if $f=0$.

By (i), $X_{z}$ is a well-defined invertible linear map. If we denote the vector $X_{z}\left(f \otimes_{A} 1_{z}\right)$ by $f(z)$, condition (iii) just says that $f(z)=0$ for every $z$ if and only if $f=0$ in $R$. In other words, $R$ is a subspace of $\mathbb{C}^{n}$-valued functions on $\Omega$. It is easy to see that

$$
f(z)=0 \Leftrightarrow X_{z}\left(f \otimes_{A} 1_{z}\right)=0 \Leftrightarrow f \otimes_{A} 1_{z}=0 \Leftrightarrow f \in R_{z} .
$$

Therefore, $R_{z}=\{f \in R \mid f(z)=0\}$, and condition (iii) is equivalent to

$$
R=\bigvee_{z \in \Omega} R_{z}^{\perp}
$$

Condition (ii) ensures that the evaluation $f \mapsto f(z)$ is bounded, i.e., $R$ is a reproducing Hilbert space. Moreover, the locally uniformly bounded assumption implies that $R$ consists of holomorphic functions. 
In fact, for any finite linear combination $\sum_{i=1}^{k} \varphi_{i} \cdot f_{i}, \varphi_{i} \in A(\Omega)$, we have $\left(\sum_{i=1}^{k} \varphi_{i} \cdot f_{i}\right)(z)=\sum_{i=1}^{k} X_{z}\left(\varphi_{i} \cdot f_{i} \otimes_{A} 1_{z}\right)=\sum_{i=1}^{k} \varphi_{i}(z) X_{z}\left(f_{i} \otimes_{A} 1_{z}\right)=\sum_{i=1}^{k} \varphi_{i}(z) e_{i}$ so $\sum_{i=1}^{k} \varphi_{i} \cdot f_{i}$ is holomorphic. On the other hand, any function $f$ in $R$ can be approximated in norm by such linear combinations. By (ii), $f(z)$ is the limit function of a sequence of locally uniformly convergent holomorphic functions, hence must be holomorphic.

We summarize these facts as a proposition:

Proposition 2.2. Any finite rank quasi-free Hilbert module $R$ over $A(\Omega)$ with generating set $\left\{f_{1}, \ldots, f_{n}\right\}$ is a reproducing Hilbert space consisting of $\mathbb{C}^{n}$-valued holomorphic functions over $\Omega$, with evaluation given by

$$
f(z)=X_{z}\left(f \otimes_{A} 1_{z}\right),
$$

where $X_{z}$ is given by Definition 2.1(ii). In particular, the generating function $f_{i}$ takes the constant value $e_{i}$.

Let $R$ and $R^{\prime}$ be two quasi-free Hilbert modules relative to generators $\left\{f_{1}, \ldots, f_{n}\right\}$ and $\left\{f_{1}^{\prime}, \ldots, f_{n}^{\prime}\right\}$ respectively. For any $z$ in $\Omega$, we can define an invertible operator $\delta_{z}: R \otimes_{A} \mathbb{C}_{z} \rightarrow R^{\prime} \otimes_{A} \mathbb{C}_{z}$ by setting $\delta_{z}\left(f_{i} \otimes_{A} 1_{z}\right)=f_{i}^{\prime} \otimes_{A} 1_{z}$. The modulus function $\mu_{\left(R, R^{\prime}\right)}$ is defined by $\mu_{\left(R, R^{\prime}\right)}(z)=\left(\delta_{z}^{*} \delta_{z}\right)^{1 / 2}$, which is a positive operator on $R \otimes_{A} \mathbb{C}_{z}$ for any $z$ in $\Omega$.

We say a function $\Psi$ belongs to $\operatorname{Hol}(\Omega)$ if for any $z, \Psi(z)$ is a linear operator on $R \otimes_{A} \mathbb{C}_{z}$ such that its matrix relative to the basis $f_{1} \otimes_{A} 1_{z}, \ldots, f_{n} \otimes_{A} 1_{z}$ varies holomorphically in $z$. In other words, $\operatorname{Hol}(\Omega)$ can be identified with the space of holomorphic $n \times n$ matrix-valued functions once we fix the frame.

In the rank one case, the Laplacian of the logarithm of the modulus function as a complex two-form is the difference of the curvatures of the line bundles associated with $R$ and $R^{\prime}$ ([5]). Hence by Theorem 1.1, the curvatures of the two associated line bundles coincide if the two modules are unitarily equivalent.

3. Unitary equivalence of quasi-free Hilbert modules. Recall that a Hilbert space $H$ of functions from $\Omega$ to $\mathbb{C}^{n}$ is a reproducing Hilbert space if the evaluation functional $\mathrm{ev}_{z}: f \mapsto f(z)$ is bounded for every $z$. In this case, $H$ admits an $n \times n$ matrix-valued kernel function $K(\cdot, \cdot)$, satisfying

$$
\langle f, K(z, \cdot) \xi\rangle_{R}=\langle f(z), \xi\rangle_{\mathbb{C}^{n}}
$$

for any $\xi$ in $\mathbb{C}^{n}$ and $z$ in $\Omega$. Here $K(z, \cdot) \xi=\mathrm{ev}_{z}^{*} \xi$ and $K(z, w)=\mathrm{ev}_{w} \mathrm{ev}_{z}^{*}$. For any $\left\{z_{i}\right\}$ in $\Omega$, and $\left\{\xi_{i}\right\},\left\{\eta_{i}\right\}$ in $\mathbb{C}^{n}$, we have the following well-known 
identity:

$$
\left\langle\sum_{i=1}^{k} K\left(z_{i}, \cdot\right) \xi_{i}, \sum_{i=1}^{k} K\left(z_{i}, \cdot\right) \eta_{i}\right\rangle_{R}=\sum_{1 \leq i, j \leq k}\left\langle K\left(z_{i}, z_{j}\right) \xi_{i}, \eta_{j}\right\rangle_{\mathbb{C}^{n}}
$$

Moreover, the finite linear sums $\sum_{i=1}^{k} K\left(z_{i}, \cdot\right) \xi_{i}$ form a dense subspace of $H$. If $H$ consists of holomorphic functions, the kernel function $K(z, w)$ is holomorphic in $w$ and anti-holomorphic in $z$.

Using the dual set of kernel functions introduced in [5], the kernel function $K(z, w)$ of a quasi-free Hilbert module $R$ can be easily calculated.

As before, let $\left\{f_{1}, \ldots, f_{n}\right\}$ be the generating set of $R$. By the Riesz Theorem, for every $z$ in $\Omega$ and $1 \leq i \leq n$, there exist $k_{z}^{1}, \ldots, k_{z}^{n}$ in $R$ satisfying the following duality:

$$
\left\langle f(z), e_{i}\right\rangle_{\mathbb{C}^{n}}=\left\langle f, k_{z}^{i}\right\rangle_{R}, \quad f \in R .
$$

In other words,

$$
k_{z}^{i}=\mathrm{ev}_{z}^{*} e_{i}
$$

for $z$ in $\Omega$ and $1 \leq i \leq n$. Consequently, $f \in R_{z}$ if and only if $f \perp k_{z}^{i}$ for every $1 \leq i \leq n$, thus $R_{z}^{\perp}=\operatorname{span}\left\{k_{z}^{1}, \ldots, k_{z}^{n}\right\}$. Moreover, regarding vectors in $\mathbb{C}^{n}$ as columns of complex numbers, we have

$$
\begin{aligned}
K(z, w) & =\left[\left\langle\mathrm{ev}_{w} \mathrm{ev}_{z}^{*} e_{j}, e_{i}\right\rangle\right]_{1 \leq i, j \leq n}=\left[\left\langle\mathrm{ev}_{z}^{*} e_{j}, \mathrm{ev}_{w}^{*} e_{i}\right\rangle\right]_{1 \leq i, j \leq n} \\
& =\left[\left\langle k_{z}^{j}, k_{w}^{i}\right\rangle\right]_{1 \leq i, j \leq n} .
\end{aligned}
$$

In particular,

$$
K(z, z)=\left[\left\langle k_{z}^{j}, k_{z}^{i}\right\rangle\right]_{1 \leq i, j \leq n},
$$

which is the transpose of the Gram matrix of the frame $\left\{k_{z}^{i}\right\}$.

Under mild conditions, operators between two reproducing Hilbert spaces commuting with coordinate operators $M_{z_{i}}$ have been completely characterized in terms of the reproducing kernels by Curto and Salinas ([3]). Following their ideas, we can give a corresponding result characterizing unitary equivalence of quasi-free Hilbert modules. We begin with a lemma on module maps.

LEMMA 3.1. Let $R$ and $R^{\prime}$ be two quasi-free Hilbert modules, and $T$ a unitary operator from $R$ to $R^{\prime}$. The following are equivalent:

(i) $T$ is a module map.

(ii) $T R_{z} \subset R_{z}^{\prime}$ for every $z \in \Omega$.

(iii) $T R_{z}^{\perp} \subset R_{z}^{\prime \perp}$ for every $z \in \Omega$.

In any case, $T R_{z}=R_{z}^{\prime}$ and $T R_{z}^{\perp}=R_{z}^{\prime \perp}$.

Proof. (i) $\Rightarrow$ (ii). This is obvious from the definition of $R_{z}$ and $R_{z}^{\prime}$. 
(ii) $\Rightarrow$ (i). We have to show that $T M_{\varphi}=M_{\varphi} T$, or equivalently, $M_{\varphi}^{*} T^{*}=$ $T^{*} M_{\varphi}^{*}$. Since $R^{\prime}=\bigvee_{z \in \Omega} R_{z}^{\prime \perp}$, it suffices to show that for any $z \in \Omega$ and $f \in R_{z}^{\perp \perp}, M_{\varphi}^{*} T^{*} f=T^{*} M_{\varphi}^{*} f$.

The condition $T R_{z} \subset R_{z}^{\prime}$ implies $T^{*} R_{z}^{\prime \perp} \subset R_{z}^{\perp}$, and it is easy to check that the action of $M_{\varphi}^{*}$ on $R_{z}^{\perp}$ and $R_{z}^{\prime \perp}$ is scalar multiplication by $\overline{\varphi(z)}$. Therefore,

$$
M_{\varphi}^{*} T^{*} f=\overline{\varphi(z)} T^{*} f=T^{*} \overline{\varphi(z)} f=T^{*} M_{\varphi}^{*} f
$$

as desired.

Note that (i) $\Leftrightarrow$ (ii) holds for any bounded operator $T$.

(i) $\Rightarrow$ (iii). Recall that $A(\Omega)_{z}=\{\varphi \in A(\Omega) \mid \varphi(z)=0\}$ and $R_{z}^{\prime}=$ $\left[A(\Omega)_{z} R^{\prime}\right]^{-}$. It suffices to show that for any $f \in R_{z}^{\perp}, \varphi \in A(\Omega)_{z}$ and $g \in R^{\prime}$,

$$
\left\langle T f, M_{\varphi} g\right\rangle=0 \text {. }
$$

Since $T$ is a unitary module map, it commutes with $M_{\varphi}$ and $M_{\varphi}^{*}$, hence

$$
\left\langle T f, M_{\varphi} g\right\rangle=\left\langle M_{\varphi}^{*} T f, g\right\rangle=\left\langle T M_{\varphi}^{*} f, g\right\rangle=\overline{\varphi(z)}\langle T f, g\rangle=0 .
$$

(iii) $\Rightarrow\left(\right.$ i). Similar arguments to those in (ii) $\Rightarrow\left(\right.$ i) imply that $T M_{\varphi}^{*}=$ $M_{\varphi}^{*} T$. Since $T$ is unitary, $T M_{\varphi}=M_{\varphi} T$. The rest is obvious.

The following theorem characterizes unitary equivalence of quasi-free Hilbert modules in terms of reproducing kernels. While our setting is slightly different from [3], the proof is essentially the same, and we only include the detailed proof of the sufficiency part for completeness.

Theorem 3.2. Let $R$ and $R^{\prime}$ be two quasi-free Hilbert modules over $A(\Omega)$ with kernel functions $K(z, w)$ and $K^{\prime}(z, w)$. $R$ and $R^{\prime}$ are unitarily equivalent if and only if there exists a matrix-valued function $\Phi$ which is invertible at every $z$ in $\Omega$ such that $K^{\prime}(z, w)=\Phi^{*}(w) K(z, w) \Phi(z)$. In this case, the unitary module map is given by $M_{\Phi^{*}}: f(\cdot) \mapsto \Phi^{*}(\cdot) f(\cdot)$.

Proof. Sufficiency: We will show that the multiplication operator $M_{\Phi^{*}}$ is a unitary module map from $R$ to $R^{\prime}$. Since $R_{z}$ and $R_{z}^{\prime}$ consist of functions vanishing at $z$, the operator $M_{\Phi^{*}}$ obviously satisfies the second condition of Lemma 3.1, so we only need to show that $M_{\Phi^{*}}$ is a well defined unitary operator.

The identity $K^{\prime}(z, w)=\Phi^{*}(w) K(z, w) \Phi(z)$ implies that for any finite set $\left\{z_{i}\right\} \subseteq \Omega$ and $\left\{\xi_{i}\right\} \subseteq \mathbb{C}^{n}, 1 \leq i \leq k$,

$$
M_{\Phi^{*}} \sum_{i=1}^{k} K\left(z_{i}, \cdot\right) \xi_{i}=\Phi^{*}(\cdot) \sum_{i=1}^{k} K\left(z_{i}, \cdot\right) \xi_{i}=\sum_{i=1}^{k} K^{\prime}\left(z_{i}, \cdot\right) \Phi^{-1}\left(z_{i}\right) \xi_{i} .
$$

Note that the right-hand side of the equation above lies in $R^{\prime}$, so $M_{\Phi^{*}}$ is a well defined operator from the subspace of finite linear combina- 
tions $\sum_{i=1}^{k} K\left(z_{i}, \cdot\right) \xi_{i}$ onto the subspace of finite linear combinations $\sum_{i=1}^{k} K^{\prime}\left(z_{i}, \cdot\right) \eta_{i}$, which are dense in $R$ and $R^{\prime}$ respectively.

Moreover,

$$
\begin{aligned}
\left\|\sum_{i=1}^{k} K^{\prime}\left(z_{i}, \cdot\right) \Phi^{-1}\left(z_{i}\right) \xi_{i}\right\|^{2} & =\sum_{1 \leq i, j \leq k}\left\langle K^{\prime}\left(z_{i}, z_{j}\right) \Phi^{-1}\left(z_{i}\right) \xi_{i}, \Phi^{-1}\left(z_{j}\right) \xi_{j}\right\rangle \\
& =\sum_{1 \leq i, j \leq k}\left\langle\Phi^{*-1}\left(z_{j}\right) K^{\prime}\left(z_{i}, z_{j}\right) \Phi^{-1}\left(z_{i}\right) \xi_{i}, \xi_{j}\right\rangle \\
& =\sum_{1 \leq i, j \leq k}\left\langle K\left(z_{i}, z_{j}\right) \xi_{i}, \xi_{j}\right\rangle=\left\|\sum_{i=1}^{k} K\left(z_{i}, \cdot\right) \xi_{i}\right\|^{2} .
\end{aligned}
$$

Now the operator $M_{\Phi^{*}}: f(\cdot) \rightarrow \Phi^{*}(\cdot) f(\cdot)$ is an isometry from a dense subspace of $R$ onto a dense subspace of $R^{\prime}$, thus admits a unique unitary extension. By continuity, the extension operator is also given by $M_{\Phi^{*}}$.

To prove our main theorem, we need to establish an elementary lemma in linear algebra. The transpose and conjugate of a matrix $A$ will be denoted by $A^{T}$ and $\bar{A}$ respectively.

Lemma 3.3. Let $H$ be a Hilbert space and $M$ be an n-dimensional subspace such that $\left\{g_{1}, \ldots, g_{n}\right\}$ is a base for $M$. If $h_{1}, \ldots, h_{n}$ are $n$ vectors in $H$ such that $\left\langle h_{i}, g_{j}\right\rangle=\delta_{i j}$ (the Kronecker symbol), then

$$
\operatorname{Gr}\left(P h_{1}, \ldots, P h_{n}\right)=\operatorname{Gr}^{*-1}\left(g_{1}, \ldots, g_{n}\right),
$$

where $P$ denotes the orthogonal projection onto $M$ and where

$$
\operatorname{Gr}\left(g_{1}, \ldots, g_{n}\right)=\left[\left\langle g_{i}, g_{j}\right\rangle\right]_{1 \leq i, j \leq n}
$$

and

$$
\operatorname{Gr}\left(P h_{1}, \ldots, P h_{n}\right)=\left[\left\langle P h_{i}, P h_{j}\right\rangle\right]_{1 \leq i, j \leq n}
$$

are the Gramians.

Proof. We first prove the following projection formula:

$$
\left(P h_{1}, \ldots, P h_{n}\right)=\left(g_{1}, \ldots, g_{n}\right) \mathrm{Gr}^{T-1}\left(g_{1}, \ldots, g_{n}\right) .
$$

To this end, we denote the right-hand side of the above equation by $\left(g_{1}^{\prime}, \ldots, g_{n}^{\prime}\right)$, which is a row of vectors in $M$. We have to check that $h_{i}-g_{i}^{\prime}$ is orthogonal to $M$, or

$$
\left\langle h_{i}-g_{i}^{\prime}, g_{j}\right\rangle=0
$$

for any $1 \leq i, j \leq n$. Equivalently,

$$
\left[\left\langle g_{i}^{\prime}, g_{j}\right\rangle\right]_{1 \leq i, j \leq n}=I .
$$


For simplicity, we introduce the following notation: for two matrices $A=$ $\left[a_{i j}\right]_{m^{\prime} \times m}$ and $B=\left[b_{i j}\right]_{m \times m^{\prime \prime}}$ with entries $a_{i j}, b_{i j}$ in $H$, define

$$
\langle A, B\rangle=\left[\sum_{k}\left\langle a_{i k}, b_{k j}\right\rangle\right]_{m^{\prime} \times m^{\prime \prime}},
$$

which is a numerical matrix. It is easy to see that if $C$ and $D$ are both numerical matrices,

$$
\langle C A, B D\rangle=C\langle A, B\rangle \bar{D} .
$$

Given vectors $f_{1}, \ldots, f_{n}$ and $f_{1}^{\prime}, \ldots, f_{n}^{\prime}$ in $H$, we have

$$
\left[\left\langle f_{i}, f_{j}^{\prime}\right\rangle\right]_{1 \leq i, j \leq n}=\left\langle\left(f_{1}, \ldots, f_{n}\right)^{T},\left(f_{1}^{\prime}, \ldots, f_{n}^{\prime}\right)\right\rangle .
$$

In particular,

$$
\operatorname{Gr}\left(f_{1}, \ldots, f_{n}\right)=\left\langle\left(f_{1}, \ldots, f_{n}\right)^{T},\left(f_{1}, \ldots, f_{n}\right)\right\rangle .
$$

Now we have

$$
\begin{aligned}
{\left[\left\langle g_{i}^{\prime}, g_{j}\right\rangle\right]_{1 \leq i, j \leq n} } & =\left\langle\left(g_{1}^{\prime}, \ldots, g_{n}^{\prime}\right)^{T},\left(g_{1}, \ldots, g_{n}\right)\right\rangle \\
& =\left\langle\left(\operatorname{Gr}^{T-1}\left(g_{1}, \ldots, g_{n}\right)\right)^{T}\left(g_{1}, \ldots, g_{n}\right)^{T},\left(g_{1}, \ldots, g_{n}\right)\right\rangle \\
& =\operatorname{Gr}^{-1}\left(g_{1}, \ldots, g_{n}\right)\left\langle\left(g_{1}, \ldots, g_{n}\right)^{T},\left(g_{1}, \ldots, g_{n}\right)\right\rangle \\
& =\operatorname{Gr}^{-1}\left(g_{1}, \ldots, g_{n}\right) \operatorname{Gr}\left(g_{1}, \ldots, g_{n}\right)=I
\end{aligned}
$$

hence the projection formula (3.3) follows.

By this formula,

$$
\begin{aligned}
\operatorname{Gr}\left(P h_{1}, \ldots, P h_{n}\right) & =\left\langle\left(P h_{1}, \ldots, P h_{n}\right)^{T},\left(P h_{1}, \ldots, P h_{n}\right)\right\rangle \\
& =\left(\operatorname{Gr}^{T-1}\left(g_{1}, \ldots, g_{n}\right)\right)^{T} \operatorname{Gr}\left(g_{1}, \ldots, g_{n}\right) \overline{\operatorname{Gr}^{T-1}\left(g_{1}, \ldots, g_{n}\right)} \\
& =\operatorname{Gr}^{*-1}\left(g_{1}, \ldots, g_{n}\right) .
\end{aligned}
$$

COROLlary 3.4. For a quasi-free Hilbert module $R$ relative to the generating set $\left\{f_{1}, \ldots, f_{n}\right\}$, we have

$$
\operatorname{Gr}\left(f_{1} \otimes_{A} 1_{z}, \ldots, f_{n} \otimes_{A} 1_{z}\right)=\operatorname{Gr}^{*-1}\left(k_{z}^{1}, \ldots, k_{z}^{n}\right),
$$

where $\left\{k_{z}^{i}\right\}$ is the dual set of kernel functions at $z$.

Proof. Since the map $R \otimes_{A} \mathbb{C}_{z} \rightarrow R_{z}^{\perp}: f \otimes_{A} 1_{z} \mapsto P_{z} f$ is unitary, we have

$$
\operatorname{Gr}\left(f_{1} \otimes_{A} 1_{z}, \ldots, f_{n} \otimes_{A} 1_{z}\right)=\operatorname{Gr}\left(P_{z} f_{1}, \ldots, P_{z} f_{n}\right) .
$$

Recall that $R_{z}^{\perp}=\operatorname{span}\left\{k_{z}^{1}, \ldots, k_{z}^{n}\right\}$, and by (3.1) we have

$$
\left\langle f_{i}, k_{z}^{j}\right\rangle_{R}=\left\langle f_{i}(z), e_{j}\right\rangle_{\mathbb{C}^{n}}=\left\langle e_{i}, e_{j}\right\rangle_{\mathbb{C}^{n}}=\delta_{i j},
$$

hence Lemma 3.3 can be applied and we are done.

Now we are ready to give the proof of our main theorem.

Proof of Theorem 1.2. Let $\left\{f_{1}, \ldots, f_{n}\right\}$ and $\left\{f_{1}^{\prime}, \ldots, f_{n}^{\prime}\right\}$ be the generating sets relative to which $R$ and $R^{\prime}$ are quasi-free. By the hypothesis of the 
theorem, we have

$$
\delta_{z}^{*} \delta_{z}=\Psi(z)^{*} \Psi(z)
$$

for a function $\Psi(z)$ in $\operatorname{Hol}(\Omega)$. Since $\delta_{z}$ is invertible, so is $\Psi(z)$, hence we have

$$
I=\left[\delta_{z} \Psi(z)^{-1}\right]^{*}\left[\delta_{z} \Psi(z)^{-1}\right] .
$$

Thus $\delta_{z} \Psi(z)^{-1}$ is unitary in the following diagram:

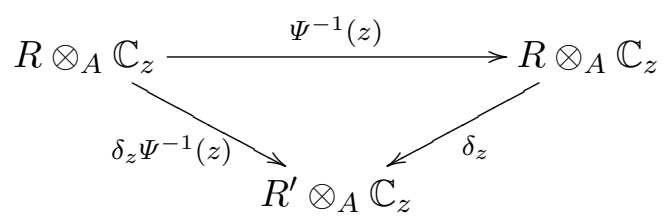

with matrix representation

$$
\begin{aligned}
\delta_{z} \Psi(z)^{-1}: R \otimes_{A} \mathbb{C}_{z} & \rightarrow R^{\prime} \otimes_{A} \mathbb{C}_{z}, \\
\left(f_{1} \otimes_{A} 1_{z}, \ldots, f_{n} \otimes_{A} 1_{z}\right) & \mapsto\left(f_{1}^{\prime} \otimes_{A} 1_{z}, \ldots, f_{n}^{\prime} \otimes_{A} 1_{z}\right) \Psi(z)^{-1} .
\end{aligned}
$$

Hence we have the following relation of Gramians:

$$
\operatorname{Gr}\left(f_{1} \otimes_{A} 1_{z}, \ldots, f_{n} \otimes_{A} 1_{z}\right)=\Psi(z)^{T-1} \operatorname{Gr}\left(f_{1}^{\prime} \otimes_{A} 1_{z}, \ldots, f_{n}^{\prime} \otimes_{A} 1_{z}\right) \overline{\Psi(z)^{-1}},
$$

where $\Psi(z)$ is a holomorphic matrix-valued function such that $\Psi(z)$ is invertible for every $z$. Appealing to Corollary 3.4 we get

$$
\operatorname{Gr}^{*-1}\left(k_{z}^{1}, \ldots, k_{z}^{n}\right)=\Psi(z)^{T-1} \mathrm{Gr}^{*-1}\left(k_{z}^{\prime 1}, \ldots, k_{z}^{\prime n}\right) \overline{\Psi(z)^{-1}} .
$$

Equivalently,

$$
\operatorname{Gr}\left(k_{z}^{1}, \ldots, k_{z}^{n}\right)=\overline{\Psi(z)} \operatorname{Gr}\left(k_{z}^{\prime 1}, \ldots, k_{z}^{\prime n}\right) \Psi(z)^{T} .
$$

Combining this with (3.2), we come to the desired identity:

$$
K(z, z)=\Psi(z) K^{\prime}(z, z) \Psi^{*}(z) .
$$

We claim the following stronger identity:

$$
K(z, w)=\Psi(w) K^{\prime}(z, w) \Psi^{*}(z) .
$$

In fact, since $\Psi(z)$ is holomorphic, the matrix-valued function $K(z, w)-$ $\Psi(w) K^{\prime}(z, w) \Psi^{*}(z)$ is holomorphic in $w$ and anti-holomorphic in $z$, and (3.5) implies that this function vanishes on the diagonal. Using Taylor expansion, it is not hard to see that such a function must vanish identically ([7, Proposition 1]), hence (3.6) holds.

Finally, we rewrite (3.6) as

$$
K^{\prime}(z, w)=\Psi(w)^{-1} K(z, w) \Psi^{-1 *}(z),
$$

so that Theorem 3.2 can be applied by taking $\Phi(z)=\Psi^{-1 *}(z)$, completing the proof. In light of Theorem 3.2, the unitary map is given by $M_{\Psi^{-1}}$ : $f(\cdot) \mapsto \Psi^{-1}(\cdot) f(\cdot)$. 
4. Equivalence of associated Hermitian vector bundles. As a complement of our main result, we characterize unitary equivalence of quasifree Hilbert modules in terms of Hermitian vector bundles. We say that two holomorphic (resp. anti-holomorphic) Hermitian vector bundles are equivalent if there exists a holomorphic (resp. anti-holomorphic) isometric bundle map between them.

Given a quasi-free Hilbert module $R$ with generator $\left\{f_{1}, \ldots, f_{n}\right\}$ and dual kernel $\left\{k_{z}^{1}, \ldots, k_{z}^{n}\right\}$, we can construct a natural bundle $\bigcup_{z \in \Omega} R_{z}^{\perp}$. As shown in [5], for any $z$ in $\Omega$ and $1 \leq i \leq n$, the map $z \mapsto k_{z}^{i}$ is anti-holomorphic in $z$, thus $\bigcup_{z \in \Omega} R_{z}^{\perp}$ is an anti-holomorphic Hermitian vector bundle such that $\left\{k_{z}^{i}\right\}$ forms an anti-holomorphic frame. This bundle, obtained as the pull-back of the anti-holomorphic map $z \mapsto R_{z}^{\perp}$ from $\Omega$ to the Grassmann manifold of $n$-dimensional subspaces of $R$, is closely related to unitary invariants of quasi-free Hilbert modules.

Using the Rigidity Theorem established in [2], one can show that two finite rank quasi-free Hilbert modules $R$ and $R^{\prime}$ are unitarily equivalent if and only if $\bigcup_{z \in \Omega} R_{z}^{\perp}$ and $\bigcup_{z \in \Omega} R_{z}^{\perp}$ are equivalent.

On the other hand, there is another vector bundle $\operatorname{Sp}(R)$ that can be associated to a quasi-free Hilbert module $R$, which is holomorphic with fibre $R \otimes_{A} \mathbb{C}_{z}$ at each $z$ in $\Omega$. This bundle $\operatorname{Sp}(R)=\bigcup_{z \in \Omega} R \otimes_{A} \mathbb{C}_{z}$ is called the spectral sheaf of $R$. Douglas and Misra proved the following result:

Proposition 4.1 ([5]). For a finite rank quasi-free Hilbert module over $A(\Omega)$, there is a unique well-defined holomorphic structure on $\operatorname{Sp}(R)$ relative to which the functions $z \mapsto k \otimes_{A} 1_{z}$ are holomorphic sections for each $k$ in $R$.

Since $\left\{f_{i} \otimes 1_{z}\right\}$ spans $R \otimes_{A} \mathbb{C}_{z}$, it is a holomorphic frame for $\operatorname{Sp}(R)$. The metric of the fibre $R \otimes_{A} \mathbb{C}_{z}$ is induced by the metric on $R_{z}^{\perp}$ in $R$ via the unitary map

$$
R \otimes_{A} \mathbb{C}_{z} \rightarrow R_{z}^{\perp}: f \otimes_{A} 1_{z} \mapsto P_{z} f .
$$

Douglas and Misra showed in [5] that this induced metric makes $\operatorname{Sp}(R)$ a holomorphic Hermitian vector bundle over $\Omega$.

Finally, we give the following geometric characterization.

TheOREM 4.2. For two finite rank quasi-free Hilbert modules $R$ and $R^{\prime}$, the following are equivalent:

(i) $R$ and $R^{\prime}$ and unitarily equivalent.

(ii) The anti-holomorphic Hermitian vector bundles $\bigcup_{z \in \Omega} R_{z}^{\perp}$ and $\bigcup_{z \in \Omega} R_{z}^{\prime \perp}$ are equivalent.

(iii) The holomorphic Hermitian vector bundles $\operatorname{Sp}(R)$ and $\operatorname{Sp}\left(R^{\prime}\right)$ are equivalent.

Proof. (i) $\Leftrightarrow($ ii). Combine the Rigidity Theorem ([2]) with Lemma 3.1. 
(ii) $\Rightarrow$ (iii). By definition, there exists an anti-holomorphic matrix-valued function $\Theta(z)$ such that for any $z$ in $\Omega$, the fibre map

$$
\begin{aligned}
\Theta(z): R_{z}^{\perp} & \rightarrow R_{z}^{\prime \perp}, \\
\left(k_{z}^{1}, \ldots, k_{z}^{n}\right) & \mapsto\left(k_{z}^{\prime 1}, \ldots, k_{z}^{\prime n}\right) \Theta(z),
\end{aligned}
$$

is isometric. Hence we have

$$
\operatorname{Gr}\left(k_{z}^{1}, \ldots, k_{z}^{n}\right)=\Theta(z)^{T} \operatorname{Gr}\left(k_{z}^{\prime 1}, \ldots, k_{z}^{\prime n}\right) \overline{\Theta(z)} .
$$

Applying Corollary 3.4, we get

$$
\operatorname{Gr}\left(f_{1} \otimes_{A} 1_{z}, \ldots, f_{n} \otimes_{A} 1_{z}\right)=\overline{\Theta(z)^{-1}} \operatorname{Gr}\left(f_{1}^{\prime} \otimes_{A} 1_{z}, \ldots, f_{n}^{\prime} \otimes_{A} 1_{z}\right) \Theta^{T-1}(z) .
$$

Therefore, the fibre map defined by

$$
\begin{aligned}
\widehat{\Theta}(z): R \otimes_{A} \mathbb{C}_{z} & \rightarrow R^{\prime} \otimes_{A} \mathbb{C}_{z}, \\
\left(f_{1} \otimes_{A} 1_{z}, \ldots, f_{n} \otimes_{A} 1_{z}\right) & \mapsto\left(f_{1}^{\prime} \otimes_{A} 1_{z}, \ldots, f_{n}^{\prime} \otimes_{A} 1_{z}\right) \Theta^{*-1}(z),
\end{aligned}
$$

is isometric. Noting that $\left\{f_{i} \otimes 1_{z}\right\}$ is a holomorphic frame, and $\Theta^{*-1}(z)$ is holomorphic since $\Theta(z)$ is anti-holomorphic, we see that the fibre maps $\widehat{\Theta}(z)$ glue to an isometric holomorphic bundle map from $\operatorname{Sp}(R)$ to $\operatorname{Sp}\left(R^{\prime}\right)$, hence $\operatorname{Sp}(R)$ and $\operatorname{Sp}\left(R^{\prime}\right)$ are equivalent.

(iii) $\Rightarrow$ (ii) follows in the same way.

REMARK 4.3. It is easy to see that if the modulus function $\mu_{\left(R, R^{\prime}\right)}$ is the absolute value of a function $\Psi$ in $\operatorname{Hol}(\Omega)$, then $\operatorname{Sp}(R)$ and $\operatorname{Sp}\left(R^{\prime}\right)$ are equivalent via the fibre map given by $\Psi(z)^{-1}$ with respect to the holomorphic frames, therefore Theorem 1.2 can be regarded as a consequence of Theorem 4.2, and this essentially relies on the Rigidity Theorem.

\section{References}

[1] X. Chen and R. G. Douglas, Localization of Hilbert modules, Michigan Math. J. 39 (1992), 443-454.

[2] M. Cowen and R. G. Douglas, Complex geometry and operator theory, Acta Math. 141 (1978), 187-261.

[3] R. E. Curto and N. Salinas, Generalized Bergman kernels and the Cowen-Douglas theory, Amer J. Math. 106 (1984), 447-488.

[4] R. G. Douglas and G. Misra, Quasi-free resolutions of Hilbert modules, Integral Equations Operator Theory 99 (2003), 435-456.

[5] —, - On quasi-free Hilbert modules, New York J. Math. 11 (2005), 547-561.

[6] R. G. Douglas and V. I. Paulsen, Hilbert Modules over Function Algebras, Pitman Res. Notes Math. Ser. 217, Longman, 1989. 
[7] M. Engliš, Density of algebras generated by Toeplitz operator on Bergman spaces, Ark. Mat. 30 (1992), 227-243.

School of Mathematics Science

Fudan University

Shanghai, 200433, China

E-mail: 051018011@fudan.edu.cn

Received January 7, 2009

Revised version May 24, 2009 